\title{
300 LONGITUDINAL PLASMA PROTEOMIC PROFILING OF NON-SMALL CELL LUNG CANCER PATIENTS UNDERGOING IMMUNE CHECKPOINT BLOCKADE-BASED THERAPY
}

${ }^{1}$ Michal Harel*, ${ }^{1}$ Coren Lahav, ${ }^{1}$ Eyal Jacob, ${ }^{1}$ Itamar Sela, ${ }^{1}$ Yehonatan Elon, ${ }^{1}$ Galit Yahalom, ${ }^{2}$ rris Kamer, ${ }^{1}$ Ofer Sharon, ${ }^{3}$ Yuval Shaked, ${ }^{4}$ Adam Dicker, ${ }^{5}$ David Carbone, ${ }^{2}$ Jair Bar. 'Oncohost LTD, Binyamina, Israel; ${ }^{2}$ Chaim Sheba Medical Center, Ramat Gan, Israel; ${ }^{3}$ Technion-Israel Institute of Technology, Haifa, Israel; ${ }^{4}$ Thomas Jefferson University, Philadelphia, PA, USA; ${ }^{5}$ The Ohio State University, Columbus, OH, USA

Background Immune checkpoint inhibitors (ICIs) have revolutionized cancer treatment by shifting the focus from the tumor to the immune system of the host. Despite durable response to ICIs, only a small proportion of non-small lung cancer (NSCLC) patients respond to this treatment. Thus, great effort is currently focused on uncovering mechanisms of resistance and identifying predictive biomarkers for outcome.

Methods Blood plasma was obtained from 143 NSCLC patients treated with ICI-based therapy at baseline and early on-treatment (following the first treatment), and the levels of approximately 800 proteins were determined using ELISAbased arrays. Bioinformatic analysis was performed in order to detect novel patterns of resistance to ICI-based therapy. To identify a signature that predicts clinical outcome, a machine learning algorithm was applied.

Results Unsupervised bioinformatic analysis of the plasma proteomic profiles classified the patients into 3 clusters with distinct clinical and biological features. Patients in cluster \#1 exhibited resistance to therapy, bone metastasis and high TNM (tumors, nodes and metastasis) staging; this cluster displayed high levels of proteins related to glycan and pyrimidine metabolism and cell-adhesion. Cluster \#2 was enriched with responders, males, and patients with low TNM staging; this cluster displayed a strong representation of desmoglein proteins. Cluster \#3 was enriched with female patients while the proteome of these patients displayed high levels of MAPK signaling related proteins. Patient clusters were largely unchanged when comparing baseline and on-treatment data, suggesting pre-existing rather than acquired resistance to therapy. A further comparison between responders and non-responders identified six significantly differentially expressed proteins comprised of both host- and tumor-related proteins, with nonresponders displaying a significant enrichment of neutrophil proteins at baseline and early-on-treatment. Notably, there was no significant difference in the neutrophil count between responders and non-responders, suggesting a functional shift in neutrophils upon treatment in non-responders. Lastly, we identified a predictive signature for response comprised of two proteins and two clinical features. The performance of the predictive signature reached an area under the curve (AUC) of the receiver operating characteristics (ROC) plot of 0.8 in an independent validation subset of the cohort, indicating a high predictive power.

Conclusions Here we performed a deep bioinformatic analysis of plasma proteome profiles of 143 NSCLC patients undergoing ICI-based therapy. Our study sheds light on underlying mechanisms of resistance to ICI-based therapy and reveals a predictive signature for response in NSCLC patients.

Ethics Approval Data and study specimens were purchased from Indivumed and Sheba medical center, approval number 0226-13-SMC (institutional review board). Participants gave informed consent before taking part. 